\title{
The cytotoxic macrolide FD-891 induces caspase-8- dependent mitochondrial release of cytochrome $c$ and subsequent apoptosis in human leukemia Jurkat cells
}

\author{
Susumu Inaba ${ }^{1}$, Tadashi Eguchi ${ }^{2}$, Atsushi Motegi ${ }^{2}$, Kazutoshi Mizoue $^{3}$, Takeo Usui ${ }^{4}$, Kazuo Nagai $^{5}$ \\ and Takao Kataoka ${ }^{1,6}$
}

The 16-membered macrolide FD-891 exerts cytotoxicity toward several cancer cell lines. In this study, we showed that FD-891 induces apoptosis in various human cancer cell lines. Human leukemia Jurkat cells were highly sensitive to FD-891, exhibiting caspase activation and mitochondrial release of cytochrome $c$ into the cytosol at early time points after exposure to FD-891. By contrast, Jurkat cells deficient in caspase-8 were resistant to FD-891-induced apoptosis and manifested little induction of cytochrome $c$ release as well as caspase-9 processing. Consistent with these results, the overexpression of the Bcl-2 family member $\mathrm{Bcl}-\mathrm{x}_{\mathrm{L}}$ or the caspase-8 modulator $\mathrm{C}-\mathrm{FLIP} \mathrm{P}_{\mathrm{L}}$ markedly prevented $\mathrm{FD}-891$-induced apoptosis. These results clearly demonstrate that FD-891 triggers caspase-8-dependent mitochondrial release of cytochrome $c$ and subsequent apoptosis in Jurkat cells.

The Journal of Antibiotics (2009) 62, 507-512; doi:10.1038/ja.2009.62; published online 10 July 2009

Keywords: apoptosis; caspase-8; cytochrome $c$; cytotoxicity; FD-891; macrolide

\section{INTRODUCTION}

Apoptosis is a physiological process that has an important role in the homeostasis of mature tissues. Caspases are a family of cysteine proteinases that are essential for apoptosis and act by cleaving selected substrates. ${ }^{1-3}$ Initiator caspase-8 is converted into active forms via dimerization and self-processing, and subsequently cleaves various substrates, such as effector caspases and BH3-only protein Bid, to produce their active forms. ${ }^{1-3}$ Truncated Bid is translocated to mitochondria where it initiates the release of pro-apoptotic proteins into the cytosol. ${ }^{4,5}$ Mitochondrial pro-apoptotic proteins, such as cytochrome $c$, are confined to intermembrane space and liberated into the cytosol in response to apoptotic stimuli. ${ }^{6,7}$ Cytochrome $c$ collaborates with adaptor protein Apaf-1 to activate initiator caspase- $9 .^{8-10}$ Effector caspases-3, -6 , and -7 are processed into their active forms by activated caspase- 9 and the activated caspases mediate the proteolytic cleavage of cellular proteins required for apoptosis. ${ }^{11}$

The cytotoxic macrolide FD-891 was originally isolated from the fermentation broth of Streptomyces graminofaciens A-8890.12,13 The absolute structure of FD-891 was determined in our earlier work (Figure 1a) ${ }^{14,15}$ and its planar structure turned out to be the same as that of BE-45653. ${ }^{16}$ The total synthesis of FD-891 was recently reported. ${ }^{17-19}$ It has been shown that FD-891 exerts cytotoxicity toward several cancer cell lines. ${ }^{12,19}$ Moreover, as regards immunosuppressive activity, FD-891 prevents cytotoxic T lymphocyte (CTL) mediated killing pathway. ${ }^{20}$ In this study, we investigated the molecular mechanism underlying the cytotoxicity of FD-891. Our present results demonstrate that FD-891 triggers caspase-8-dependent mitochondrial release of cytochrome $c$ and subsequent apoptosis in a human leukemia cell line highly sensitive to FD-891.

\section{MATERIALS AND METHODS \\ Cells}

Human lung carcinoma A549 cells, human fibrosarcoma HT-1080 cells and human rhabdomyosarcoma Kym-1 cells were obtained from the Health Science Research Resources Bank (Osaka, Japan). Caspase-8-deficient Jurkat cell clone (I9.2), ${ }^{21}$ c-FLIP ${ }_{\mathrm{L}}$-transfected Jurkat cell clone (JFL2), ${ }^{22}$ and Bcl- $\mathrm{x}_{\mathrm{L}}$-transfected Jurkat cell clone (Jurkat-Bcl- $\left.\mathrm{x}_{\mathrm{L}} \# 1\right)^{23}$ were described previously. Human cancer cell lines were maintained in RPMI-1640 medium (Invitrogen, Carlsbad, CA, USA) supplemented with $10 \%(\mathrm{v} / \mathrm{v})$ heat-inactivated fetal calf serum (JRH Bioscience, Lenexa, KS, USA), penicillin $\mathrm{G}\left(100 \mathrm{U} \mathrm{ml}^{-1}\right)$ (Sigma-Aldrich, St Louis, MO, USA) and streptomycin $\left(100 \mu \mathrm{g} \mathrm{m}^{-1}\right)$ (Sigma-Aldrich).

\section{Reagents}

FD-891 was purified from the fermentation broth of S. graminofaciens A-8890 as previously described. ${ }^{12}$ Doxorubicin, etoposide and vincristine were purchased from Sigma-Aldrich.

${ }^{1}$ Center for Biological Resources and Informatics, Tokyo Institute of Technology, Nagatsuta-cho, Midori-ku, Yokohama, Japan; ${ }^{2}$ Department of Chemistry and Materials Science, Tokyo Institute of Technology, O-okayama, Meguro-ku, Tokyo, Japan; ${ }^{3}$ Taisho Pharmaceutical Co. Ltd., Yoshino-cho, Kita-ku, Saitama-shi, Saitama, Japan; ${ }^{4}$ Graduate School of Life and Environmental Sciences, University of Tsukuba, Tennodai, Tsukuba, Ibaraki, Japan; ${ }^{5}$ Department of Biological Chemistry, Chubu University, Matsumoto-cho, Kasugai, Aichi, Japan and ${ }^{6}$ Department of Applied Biology, Kyoto Institute of Technology, Matsugasaki, Sakyo-ku, Kyoto, Japan

Correspondence: Professor T Kataoka, Department of Applied Biology, Kyoto Institute of Technology, Matsugasaki, Sakyo-ku, Kyoto 606-8585, Japan.

E-mail: takao.kataoka@kit.ac.jp

Received 3 June 2009; revised 8 June 2009; accepted 18 June 2009; published online 10 July 2009 


\section{Antibodies}

Antibodies to caspase-3 (H-277; Santa Cruz Biotechnology, Santa Cruz, CA, USA), caspase-6 (3E8; Medical \& Biological Laboratories (MBL), Nagoya, Japan), caspase-7 (11E4; Sigma-Aldrich), caspase-8 (5F7; MBL), caspase-9 (5B4; MBL), cytochrome $c$ (7H8.2C12; BD Biosciences, Franklin Lakes, NJ, USA), and PARP (C-2-10; Sigma-Aldrich) were commercially obtained.

\section{Assay for cell viability}

Cells were pulsed with $500 \mu \mathrm{g} \mathrm{ml}^{-1}$ of 3-(4,5-dimethylthiazol-2-yl)-2,5-diphenyl tetrazolium bromide (MTT) for $4 \mathrm{~h}$. MTT formazan was solubilized with $10 \%$

a

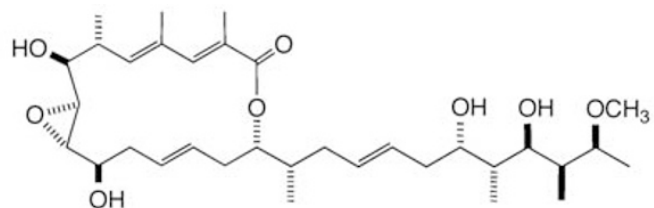
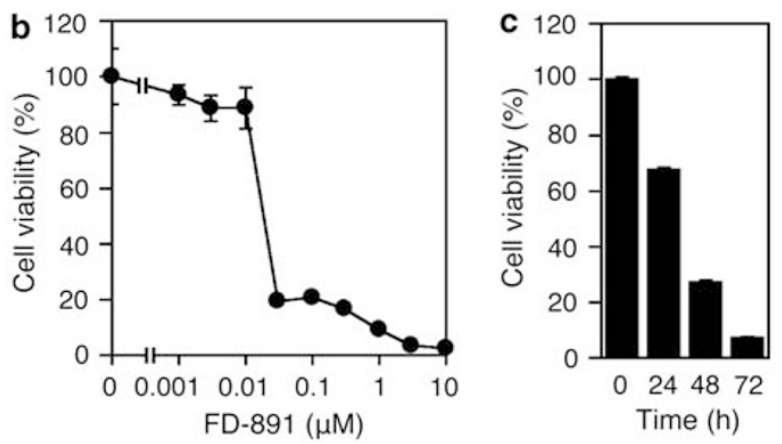

Figure 1 FD-891 inhibits cell proliferation. (a) Structure of FD-891. (b and c) Jurkat cells were incubated with various concentrations of FD-891 for $72 \mathrm{~h}$ (b). Jurkat cells were incubated with FD-891 $(1 \mu \mathrm{m})$ for the indicated times (c). Cell viability (\%) was measured by MTT assay. Data points represent means \pm s.d. of triplicate cultures. sodium dodecyl sulfate (SDS) overnight. Absorbance at $595 \mathrm{~nm}$ was measured with a Model 680 microplate reader (Bio-Rad Laboratories, Hercules, CA, USA). Cell viability (\%) was calculated as [(experimental absorbance-background absorbance)/(control absorbance -background absorbance) $] \times 100$.

\section{Assay for apoptosis}

Cells were fixed with PBS containing $4 \%$ paraformaldehyde at $4{ }^{\circ} \mathrm{C}$ overnight, and then stained with $300 \mu \mathrm{m}$ Hoechst 33342 (Calbiochem, San Diego, CA, USA). Nuclear morphology was observed under a fluorescence light microscope (Axiovert $200 \mathrm{M}$; Carl Zeiss, Jena, Germany). Apoptotic cells (\%) were calculated as (condensed nuclei/total nuclei) $\times 100$.

\section{Preparation of cell lysates}

Cells were washed with PBS and lysed in Triton X-100 lysis buffer consisting of $50 \mathrm{~mm}$ Tris- $\mathrm{HCl}$ (pH 7.4), 1\% Triton X-100, 2 mм DTT, 2 mм sodium orthovanadate, and the protease inhibitor mixture Complete (Roche Diagnostics,

Table 1 Effect of FD-891 on proliferation of human cancer cell lines

\begin{tabular}{llc}
\hline Cell line & Source & IC $_{50}$ (nM) \\
\hline A549 & Lung carcinoma & $310 \pm 70$ \\
HeLa & Cervix adenocarcinoma & $140 \pm 70$ \\
HL-60 & Acute promyelocytic leukemia & $35 \pm 29$ \\
HT-1080 & Fibrosarcoma & $130 \pm 70$ \\
Jurkat & Acute T cell leukemia & $32 \pm 24$ \\
K-562 & chronic myelogenous leukemia & $1000 \pm 710$ \\
Kym-1 & Rhabdosarcoma & $250 \pm 80$ \\
MCF7 & Breast adenocarcinoma & $220 \pm 100$ \\
Raji & Burkitt's lymphoma & $170 \pm 140$ \\
THP-1 & Acute monocytic lymphoma & $30 \pm 20$ \\
U-937 & Histiocytic lymphoma & $41 \pm 34$ \\
\hline
\end{tabular}

Human cancer cell lines were incubated with serial dilution of FD-891 for 3 days. Cell viability (\%) was measured by MTT assay. The $\mathrm{IC}_{50}$ values (mean \pm s.d.) were calculated from three to four independent experiments.
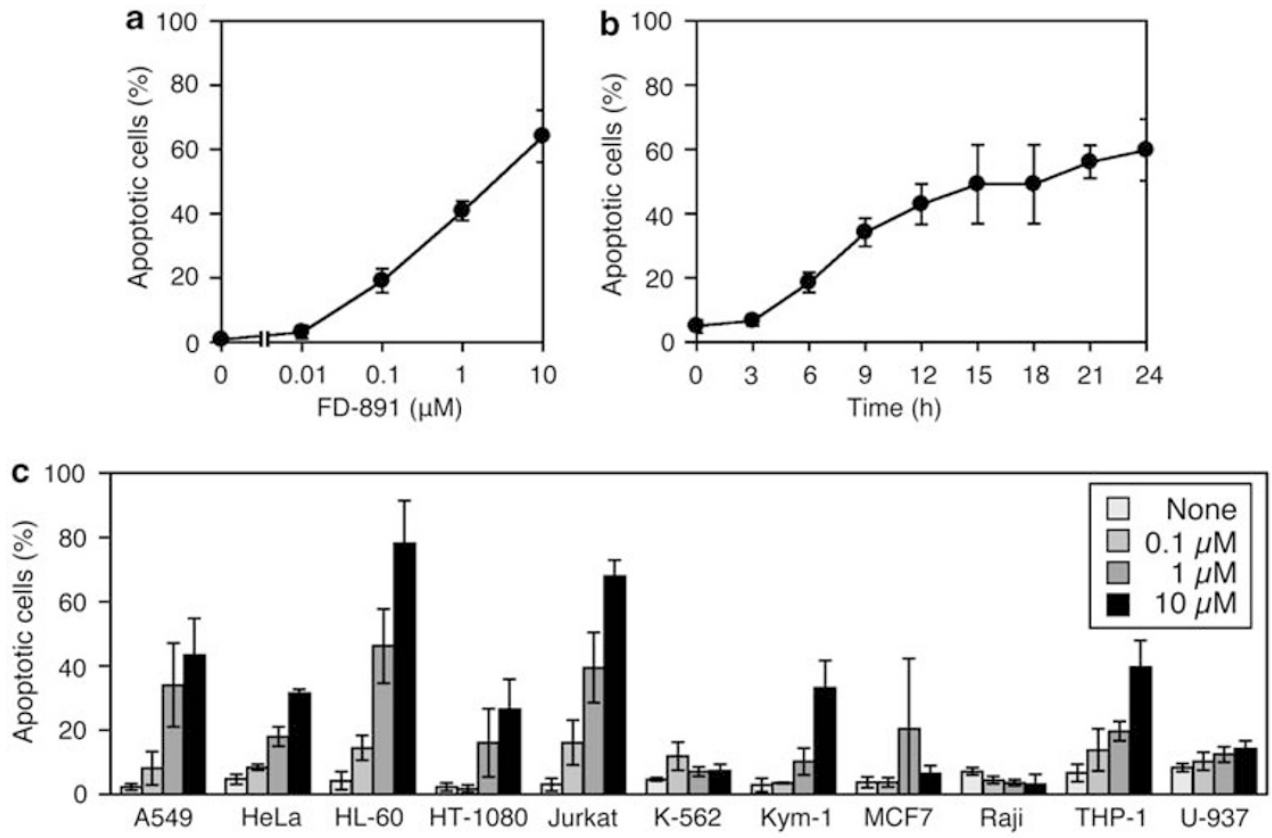

Figure 2 FD-891 induces apoptosis. (a and $\mathbf{b})$ Jurkat cells were incubated with various concentrations of FD-891 for $24 \mathrm{~h}$ (a). Jurkat cells were incubated with FD-891 $(1 \mu \mathrm{m})$ for the indicated times (b). Apoptotic cells (\%) were measured by Hoechst 33342 staining. Data points represent means \pm s.d. of triplicate determinations. (c) Human cancer cell lines were incubated with various concentrations of FD-891 for $24 \mathrm{~h}$. Apoptotic cells (\%) were measured by Hoechst 33342 staining. The results are expressed as means \pm s.d. of three independent experiments. 
Mannheim, Germany). Postnuclear lysates were collected as supernatants by centrifugation $(10000 \mathrm{~g}, 5 \mathrm{~min})$. Alternatively, cells were washed with PBS and lysed with digitonin lysis buffer (10 mM Hepes- $\mathrm{KOH}(\mathrm{pH} 7.2), 100 \mu \mathrm{M}$ digitonin, $250 \mathrm{~mm}$ sucrose, $1 \mathrm{~mm}$ DTT, 5 mм EGTA, $2 \mathrm{~mm} \mathrm{MgCl}_{2}, 50 \mathrm{~mm} \mathrm{NaCl}$ and the protease inhibitor mixture Complete) on ice for $15 \mathrm{~min}$. After centrifugation $(10000 \mathrm{~g}, 5 \mathrm{~min})$, cell lysates containing the cytosol were recovered as supernatants. Pellets were washed once with digitonin lysis buffer and treated with Triton X-100 lysis buffer on ice for $15 \mathrm{~min}$. After centrifugation $(10000 \mathrm{~g}$, $5 \mathrm{~min}$ ), cell lysates containing the mitochondria were collected as supernatants (mitochondrial fraction).

\section{Western blotting}

Protein samples ( $30 \mu \mathrm{g}$ per lane) were separated by SDS-PAGE and transferred onto Hybond-ECL nitrocellulose membranes (GE Healthcare, Piscataway, NJ, USA). The transferred membranes were stained with Ponceau $S$ and checked for equal loading of proteins before antibody reaction. The membranes were incubated with primary antibodies and then with horseradish peroxidaseconjugated secondary antibodies (Jackson ImmunoResearch, West Grove, PA, USA), followed by analysis using ECL western blotting detection reagents (GE Healthcare).

\section{RESULTS}

FD-891 inhibits proliferation of human cancer cell lines It has been shown that FD-891 exerts cytotoxicity toward several cancer cell lines. ${ }^{12,19}$ To investigate the inhibition specificity of FD-891 on cancer cell proliferation, various human cancer cell lines were incubated in the presence of FD-891 for $72 \mathrm{~h}$ and cell viability was measured by the MTT assay. Although FD-891 prevented cell proliferation at $\mathrm{IC}_{50}$ values of 130 to $1000 \mathrm{~nm}$ in most of the cancer cell lines, four leukemia cell lines (HL-60, Jurkat, THP-1 and U-937) were highly sensitive to FD-891 at IC $_{50}$ values of approximately 30 to $40 \mathrm{~nm}$ (Table 1). In Jurkat cells, FD-891 decreased cell viability by more than $80 \%$ at concentrations higher than $100 \mathrm{~nm}$ when incubated for $72 \mathrm{~h}$ (Figure 1b). Kinetic studies revealed that FD-891 gradually reduced cell viability during incubation for 24 to $72 \mathrm{~h}$ (Figure 1c).

\section{FD-891 induces apoptosis of human cancer cell lines}

To investigate whether FD-891 is able to induce apoptosis, Jurkat cells were incubated with various concentrations of FD-891 for $24 \mathrm{~h}$ and nuclear morphological changes were analyzed by fluorescence microscopy after Hoechst 33342 staining. FD-891 at concentrations higher than $100 \mathrm{~nm}$ was found to induce nuclear condensation and fragmentation characteristic of apoptosis within $24 \mathrm{~h}$ (Figure 2a). Kinetic studies revealed that FD-891 initiated apoptosis within $6 \mathrm{~h}$ and that the number of apoptotic cells steadily increased during $24 \mathrm{~h}$ incubation (Figure 2b). Various human cancer cell lines were also found to undergo apoptosis upon treatment with FD-891. FD-891 was able to induce apoptosis very strongly in two leukemia cell lines (that is, Jurkat and HL-60), whereas it induced moderate-to-weak apoptosis in a

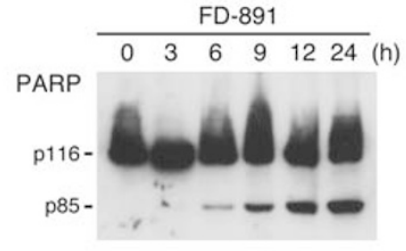

b
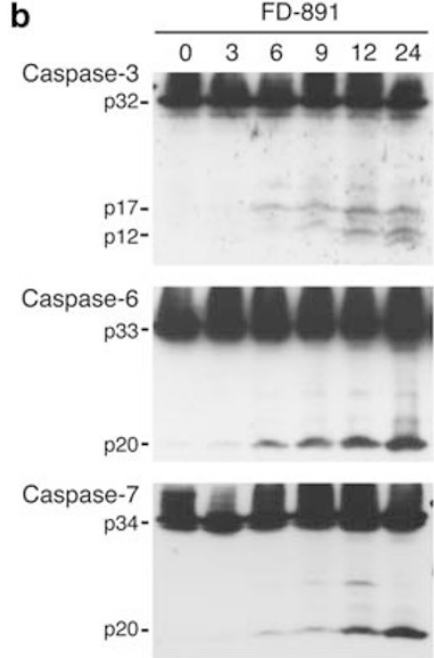

C

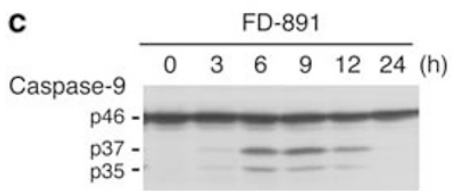

d

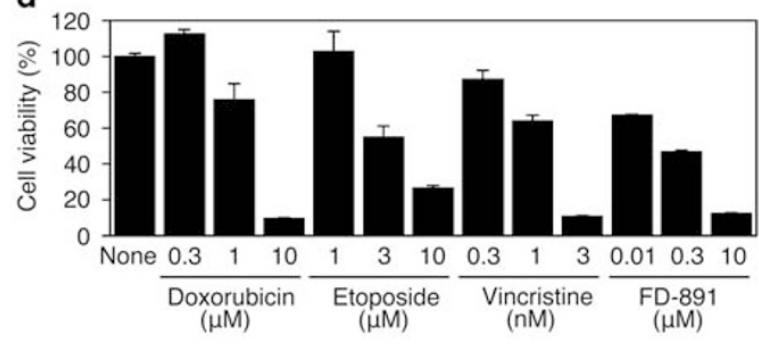

e

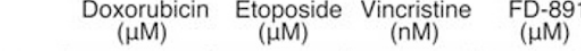

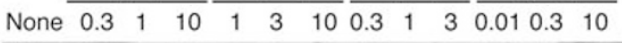

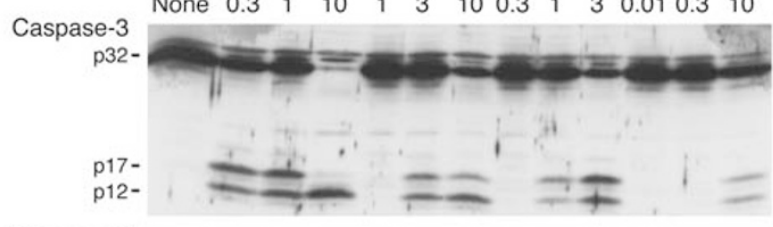

Caspase- 6

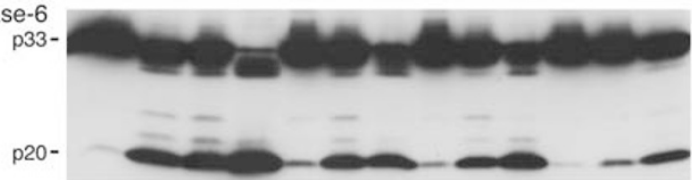

Caspase-7

p34-

p20-

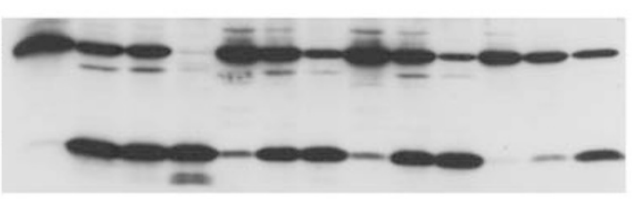

Figure 3 FD-891 induces activation of effector caspases. (a-c) Jurkat cells were incubated with FD-891 ( $1 \mu \mathrm{m}$ ) for the indicated times. The expression of PARP (a), caspases-3, -6, -7 (b), and caspase-9 (c) was analyzed by western blotting. (d) Jurkat cells were incubated with various concentrations of doxorubicin, etoposide, vincristine, and FD-891 for $24 \mathrm{~h}$. Cell viability (\%) was measured by MTT assay. The results are means \pm s.d. of triplicate cultures. (e) Jurkat cells were incubated with various concentrations of doxorubicin, etoposide, vincristine and FD-891 for $24 \mathrm{~h}$. The expression of caspases-3, -6 and -7 was analyzed by western blotting. 
the other cancer cell lines (Figure 2c). Therefore, the results suggest that the induction of apoptosis is correlated with the inhibitory effect of FD-891 on cell proliferation at least in Jurkat and HL-60 cells. However, non-apoptotic cells harboring two nuclei apparently accumulated in FD-891-treated cells (unpublished observation). Therefore, as previously reported, ${ }^{19}$ it seems that FD-891 induces cell cycle arrest at the $\mathrm{G}_{2} / \mathrm{M}$ phase, and this biological effect may also contribute to its inhibitory effect on cell proliferation. In this study, the molecular mechanism underlying FD-891-induced apoptosis in Jurkat cells was further addressed.

\section{FD-891 induces activation of caspases}

Poly(ADP-ribose)polymerase (PARP) is a nuclear protein that is processed by effector caspases during apoptosis. ${ }^{24}$ When Jurkat cells were incubated with FD-891, full-length PARP $(116 \mathrm{kDa})$ was processed into the cleaved form $(85 \mathrm{kDa})$ during 6 to $24 \mathrm{~h}$ incubation (Figure 3a). Likewise, caspase-3, caspase- 6 and caspase- 7 were processed into their active forms in FD-891-treated cells with similar kinetics (Figure 3b). Initiator caspase-9 was also processed into p35 and p37 fragments at earlier time points than other effector caspases when Jurkat cells were exposed to FD-891 (Figure 3c). We previously reported that chemotherapeutic agents, such as doxorubicin, etoposide and vincristine, induce apoptosis in Jurkat cells. ${ }^{25}$ Similar to FD-891, doxorubicin, etoposide and vincristine were able to induce the processing of caspase- 3 , caspase- 6 and caspase- 7 into their active forms (Figure 3e). However, at the concentrations that reduced cell viability to approximately $50 \%$ within $24 \mathrm{~h}$, these chemotherapeutic drugs were able to induce the activation of effector caspases more effectively than FD-891 (Figures 3d and e). Thus, the results suggest that FD-891 may induce caspase activation in a manner distinct from doxorubicin, etoposide and vincristine.

\section{FD-891 induces mitochondrial release of cytochrome $c$}

Mitochondria has an essential role in the induction of apoptosis by releasing pro-apoptotic proteins, such as cytochrome $c$, into the cytosol. ${ }^{6,7}$ Upon treatment with FD-891, cytochrome $c$ was released from the mitochondria into the cytosol in Jurkat cells (Figure 4a). The Bcl-2 family of proteins regulates cytochrome $c$ release and subsequent induction of apoptosis. ${ }^{6,7}$ The overexpression of $\mathrm{Bcl}-\mathrm{x}_{\mathrm{L}}$ markedly inhibited apoptosis induced by FD-891 (Figure 4b). These results suggest that the mitochondrial release of cytochrome $c$ is required for FD-891-induced apoptosis.

\section{Caspase-8 is required for mitochondrial release of cytochrome $c$ during FD-891-induced apoptosis}

Caspase- 8 is an initiator caspase that undergoes self-processing into its active forms in response to various stimuli. ${ }^{1-3}$ In Jurkat cells, caspase-8 was processed into p43/p41 fragments during FD-891-induced apoptosis (Figure 5a). However, in contrast to Fas ligand (FasL) stimulation, p18 large fragments were barely detectable in FD-891-treated cells (Figure 5a). To address if caspase- 8 is required for FD-891induced apoptosis, Jurkat cells deficient in caspase- 8 were compared with wild-type cells for the induction of apoptosis upon exposure to FD-891. Caspase-8-deficient cells manifested resistance to FD-891induced apoptosis (Figure 5b). The overexpression of the caspase- 8 modulator c-FLIP $_{\mathrm{L}}$ also conferred resistance to $\mathrm{FD}-891$-induced apoptosis in Jurkat cells (Figure 5c), supporting the notion that caspase-8 is required for FD-891-induced apoptosis. In caspase-8deficient cells, FD-891 barely induced the release of cytochrome $c$ into the cytosol (Figure 5d). Consistently, caspase- 9 processing was also inhibited in caspase-8-deficient cells treated with FD-891 (Figure 5e). a
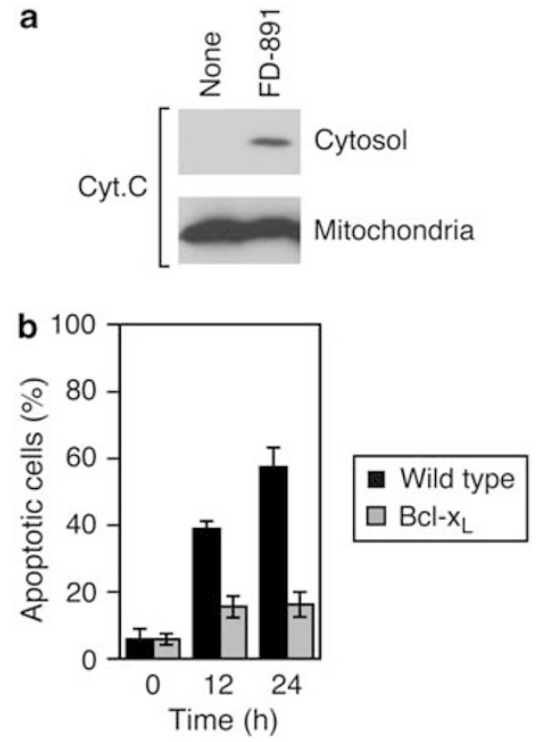

Figure 4 FD-891 induces mitochondrial release of cytochrome $c$. (a) Jurkat cells were incubated with FD-891 $(1 \mu \mathrm{M})$ for $12 \mathrm{~h}$. Cell lysates containing the cytosol and the mitochondria were prepared. The expression of cytochrome $c$ was analyzed by western blotting. (b) Wild-type Jurkat cells (black bars) and Bcl-xL-transfected Jurkat cell clone (gray bars) were incubated with FD-891 $(1 \mu \mathrm{m})$ for the indicated times. Apoptotic cells (\%) were measured by Hoechst 33342 staining. The results are expressed as means \pm s.d. of triplicate determinations.

Together, the results demonstrate that caspase- 8 is required for the release of cytochrome $c$ to the cytosol and the subsequent activation of caspase-9.

\section{DISCUSSION}

FD-891 was found to induce apoptosis in several human cancer cell lines. In Jurkat cells that were highly sensitive to FD-891, the activation of caspases as well as the mitochondrial release of cytochrome $c$ to the cytosol was induced at early time points after treatment with FD-891. Unlike wild-type Jurkat cells, FD-891 barely induced the activation of caspase- 9 and the mitochondrial release of cytochrome $c$ in caspase-8deficient Jurkat cells. The overexpression of $\mathrm{Bcl}-\mathrm{x}_{\mathrm{L}}$ or $\mathrm{c}-\mathrm{FLIP}_{\mathrm{L}}$ rendered Jurkat cells resistant to FD-891-induced apoptosis. Thus, our present results clearly demonstrate that FD-891 induces caspase-8-dependent mitochondrial release of cytochrome $c$ to the cytosol and subsequent apoptosis in Jurkat cells.

It has been shown that FD-891 inhibits cell proliferation in cancer cell lines. ${ }^{12,19}$ Consistent with these findings, we have shown that FD891 is able to inhibit cell proliferation in many human cancer cell lines at $\mathrm{IC}_{50}$ values of 0.03 to $1 \mu \mathrm{m}$. Human leukemia cell lines, such as Jurkat cells and HeLa cells, were highly sensitive to FD-891 and underwent rapid apoptosis upon treatment with FD-891. However, in some cancer cell lines, FD-891 barely induced apoptosis even at $10 \mu \mathrm{M}$, suggesting that the FD-891-induced apoptosis is cell typespecific. It has been shown that FD-891 induces accumulation of cancer cells at the $\mathrm{G}_{2} / \mathrm{M}$ phase during the cell cycle. ${ }^{19}$ Therefore, it seems likely that FD-891 predominantly induces cell cycle arrest, thereby inhibiting cell proliferation in cancer cell lines refractory to apoptosis.

Chemotherapeutic drugs, such as doxorubicin, etoposide and vincristine, are known to induce caspase-dependent apoptosis in various types of cancer cells. We previously showed that the over- 
a
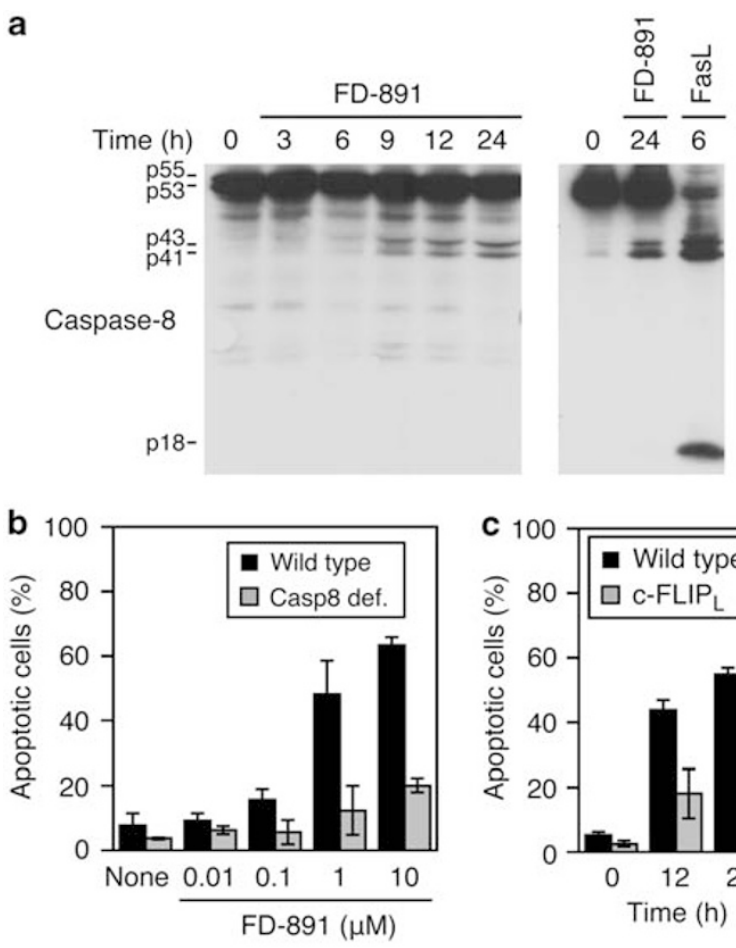

d

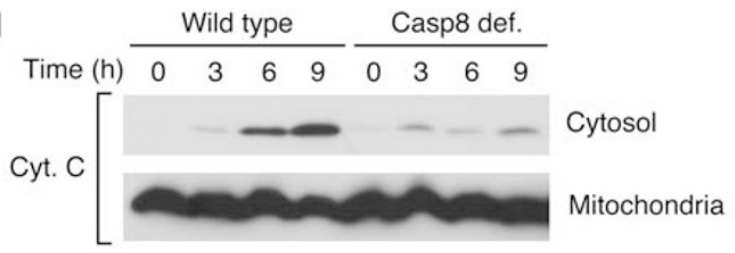

e

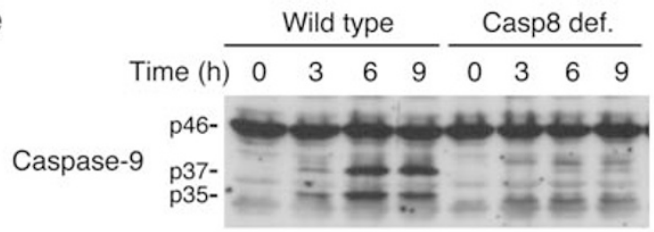

Figure 5 Caspase- 8 is required for cytochrome $c$ release and caspase- 9 activation during FD-891-induced apoptosis. (a) Jurkat cells were incubated with or without FD-891 $(1 \mu \mathrm{M})$ or FasL $\left(200 \mathrm{ng} \mathrm{m}^{-1}\right)$ for the indicated times. The expression of caspase- 8 was analyzed by western blotting. (b) Wild-type Jurkat cells (black bars) and caspase-8-deficient Jurkat cells (gray bars) were incubated with various concentrations of FD-891 for $24 \mathrm{~h}$. Apoptotic cells (\%) were measured by Hoechst 33342 staining. The results are means \pm s.d. of triplicate determinations. (c) Wild-type Jurkat cells (black bars) and C-FLIPL-transfected Jurkat cell clone JFL2 (gray bars) were incubated with FD-891 $(1 \mu \mathrm{m})$ for the indicated times. Apoptotic cells (\%) were measured by Hoechst 33342 staining. The results are means \pm s.d. of triplicate determinations. (d) Wild-type Jurkat cells and caspase-8-deficient Jurkat cells were incubated with FD-891 $(1 \mu \mathrm{m})$ for the indicated times. Cell lysates containing the cytosol and the mitochondria were prepared. The expression of cytochrome $c$ was analyzed by western blotting. (e) Wild-type Jurkat cells and caspase-8-deficient Jurkat cells were incubated with FD$891(1 \mu \mathrm{m})$ for the indicated times. The expression of caspase- 9 was analyzed by western blotting.

expression of c-FLIP $\mathrm{L}_{\mathrm{L}}$ does not prevent apoptosis induced by doxorubicin, etoposide and vincristine in Jurkat cells, ${ }^{25}$ indicating that caspase- 8 is dispensable for apoptosis induced by these chemotherapeutic drugs. However, it seems that caspase- 8 activation is required for FD-891-induced apoptosis, as c-FLIP ${ }_{\mathrm{L}}$ overexpression or caspase-8 deficiency markedly prevented FD-891-induced apoptosis. Therefore, as the main apoptosis-signaling pathway, FD-891 is likely to induce the mitochondrial release of cytochrome $c$ as well as caspase-9 processing through caspase- 8 activation.

In addition to the cytotoxicity toward cancer cells, we have previously shown that FD-891 prevents the interaction between CTLs and target cells, thereby inhibiting CTL-mediated killing pathways. ${ }^{20}$ Structurally related 16 - and 18 -membered macrolides (bafilomycin $A_{1}$ and concanamycin $A$, respectively), known as inhibitors of vacuolar-type $\mathrm{H}^{+}$-ATPase, manifested inhibitory profiles different from FD-891 in the CTL-mediated killing pathway, ${ }^{26}$ in agreement with the fact that FD-891 does not affect vacuolar acidification. ${ }^{20}$ The mechanism of action of FD-891 is also distinct from that of known chemotherapeutic drugs (doxorubicin, etoposide and vincristine). To understand anti-cancer and immunosuppressive activities at the molecular level, the molecular target(s) of FD-891 should be identified.

\section{ACKNOWLEDGEMENTS}

This work was supported by a Grant-in-Aid for Scientific Research from the Ministry of Education, Culture, Sports, Science and Technology (MEXT), Japan.

1 Riedl, S. J. \& Shi, Y. Molecular mechanisms of caspase regulation during apoptosis. Nat. Rev. Mol. Cell Biol. 5, 897-907 (2004).

2 Siegel, R. M. Caspases at the crossroads of immune-cell life and death. Nat. Rev. Immunol. 6, 308-317 (2006).

3 Krammer, P. H., Arnold, R. \& Lavrik, I. N. Life and death in peripheral T cells. Nat. Rev. Immunol. 7, 532-542 (2007)

4 Luo, X., Budihardjo, I., Zou, H., Slaughter, C. \& Wang, X. Bid, a Bcl2 interacting protein, mediates cytochrome $c$ release from mitochondria in response to activation of cell surface death receptors. Cell 94, 481-490 (1998).

$5 \mathrm{Li}, \mathrm{H} .$, Zhu, H., Xu, C. \& Yuan, J. Cleavage of BID by caspase 8 mediates the mitochondrial damage in the Fas pathway of apoptosis. Cell 94, 491-501 (1998).

6 Chipuk, J. E., Bouchier-Hayes, L. \& Green, D. R. Mitochondrial outer membrane permeabilization during apoptosis: the innocent bystander scenario. Cell Death Differ. 13, 1396-1402 (2006).

7 Garrido, C. et al. Mechanisms of cytochrome $c$ release from mitochondria. Cell Death Differ. 13, 1423-1433 (2006).

8 Pop, C., Timmer, J., Sperandio, S. \& Salvesen, G. S. The apoptosome activates caspase9 by dimerization. Mol. Cell 22, 269-275 (2006).

9 Schafer, Z. T. \& Kornbluth, S. The apoptosome: physiological, developmental, and pathological modes of regulation. Dev. Cell 10, 549-561 (2006).

10 Bao, Q. \& Shi, Y. Apoptosome: a platform for the activation of initiator caspases. Cell Death Differ. 14, 56-65 (2007).

11 Taylor, R. C., Cullen, S. P. \& Martin, S. J. Apoptosis: controlled demolition at the cellular level. Nat. Rev. Mol. Cell Biol. 9, 231-241 (2008).

12 Seki-Asano, M. et al. Isolation and characterization of new 18-membered macrolides FD-891 and FD-892. J. Antibiot. 47, 1226-1233 (1994).

13 Seki-Asano, M., Tsuchida, Y., Hanada, K. \& Mizoue, K. Structures of new 18-membered macrolides FD-891 and FD-892. J. Antibiot. 47, 1234-1241 (1994).

14 Eguchi, T. et al. Stereostructure of a novel cytotoxic 18-membered macrolactone antibiotic FD-891. Org. Lett. 4, 3383-3386 (2002).

15 Eguchi, T., Yamamoto, K., Mizoue, K. \& Kakinuma, K. Structure revision of FD-891, a 16-membered macrolide antibiotic. J. Antibiot. 57, 156-157 (2004).

16 Ogawa, H., Nakajima, S., Suzuki, H., Ojiri, K. \& Suda, H. Antitumor agent BE-45653 manufacture with Streptomyces. Jpn. Kokai Tokkyo Koho 09087285 (1997).

17 Crimmins, M. T. \& Caussanel, F. Enantioselective total synthesis of FD-891. J. Am. Chem. Soc. 128, 3128-3129 (2006).

18 García-Fortanet, J., Murga, J., Carda, M. \& Marco, J. A. Stereoselective synthesis of the cytotoxic macrolide FD-891. Org. Lett. 8, 2695-2698 (2006).

19 García-Fortanet, J. et al. The total synthesis and biological properties of the cytotoxic macrolide FD-891 and its non-natural (Z)-C12 isomer. Chem. Eur. J. 13, 5060-5074 (2007).

20 Kataoka, T. et al. FD-891, a structural analogue of concanamycin A that does not affect vacuolar acidification or perforin activity, yet potently prevents cytotoxic $T$ lymphocytemediated cytotoxicity through the blockage of conjugate formation. Immunology 100, 170-177 (2000).

21 Juo, P., Kuo, C. J., Yuan, J. \& Blenis, J. Essential requirement for caspase-8/FLICE in the initiation of the Fas-induced apoptotic cascade. Curr. Biol. 8, 1001-1008 (1998). 
22 Irmler, M. et al. Inhibition of death receptor signals by cellular FLIP. Nature 388, 190-195 (1997).

23 Kadohara, K. et al. Acetoxycycloheximide (E-73) rapidly induces apoptosis mediated by the release of cytochrome $c$ via activation of $\mathrm{c}$-Jun $\mathrm{N}$-terminal kinase. Biochem. Pharmacol. 69, 551-560 (2005).

24 Nicholson, D. W. Caspase structure, proteolytic substances, and function during apoptotic cell death. Cell Death Differ. 6, 1028-1042 (1999).
25 Kataoka, T. et al. FLIP prevents apoptosis induced by death receptors but not by perforin/granzyme B, chemotherapeutic drugs, and gamma irradiation. J. Immunol. 161, 3936-3942 (1998).

26 Kataoka, T. et al. Identification of low molecular weight probes on perforin- and Fasbased killing mediated by cytotoxic T lymphocytes. Biosci. Biotech. Biochem. 60, 1726-1728 (1996). 